\title{
Development of Non-Immunosuppressive FK506 Derivatives as Antifungal and Neurotrophic Agents
}

\author{
Jin A Jung and Yeo Joon Yoon* \\ Department of Chemistry and Nanoscience, Ewha Womans University, Seoul 03760, Republic of Korea
}

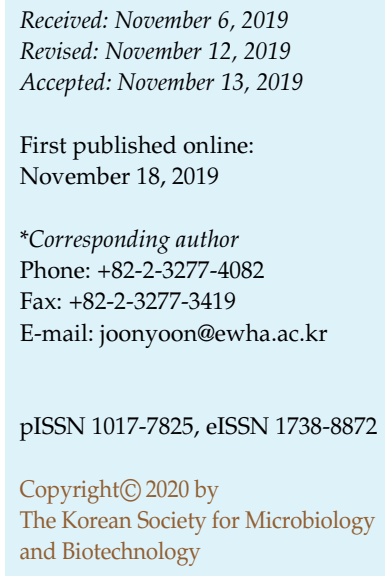

\begin{abstract}
FK506, also known as tacrolimus, is a clinically important immunosuppressant drug and has promising therapeutic potentials owing to its antifungal, neuroprotective, and neuroregenerative activities. To generate various FK506 derivatives, the structure of FK506 has been modified by chemical methods or biosynthetic pathway engineering. Herein, we describe the mode of the antifungal action of FK506 and the structure-activity relationship of FK506 derivatives in the context of immunosuppressive and antifungal activities. In addition, we discuss the neurotrophic mechanism of FK506 known to date, along with the neurotrophic FK506 derivatives with significantly reduced immunosuppressive activity. This review suggests the possibility to generate novel FK506 derivatives as antifungal as well as neuroregenerative/neuroprotective agents.
\end{abstract}

Keywords: FK506, biosynthesis, antifungal activity, neurotrophic activity

\section{Introduction}

FK506, an FDA-approved immunosuppressant, is a 23membered macrocyclic polyketide and has been used to prevent organ transplant rejection. In 1984, FK506 was first isolated from Streptomyces tsukubanesis No. 9993 found in the soil at Tsukuba, Japan [1-3]. Since then, several Streptomyces species have been discovered as FK506 producers [4-6].

Previous studies reported that FK506 exhibited various therapeutic activities, such as antifungal [7, 8], neuroprotective [9], neuroregenerative [10], and immunosuppressive activities $[1,2]$. The activity of FK506 immunosuppression results from inhibition of T-cell proliferation. FK506 interacts with an FK506-binding protein (FKBP12) to form the FKBP12-FK506 complex, which inhibits calcineurin (CaN). The resulting FKBP12-FK506-CaN complex suppresses interleukin-2 (IL-2) production and prevents T-cell activation $[11,12]$. The mechanism of antifungal action of FK506 is very similar to its immunosuppression mechanism. The CaN pathway plays essential roles in the growth and pathogenesis of fungal pathogens, and thus, the inhibition of fungal $\mathrm{CaN}$ can lead to the antifungal activity [8]. In contrast, neuronal property of FK506 may not be directly related to CaN; however, FKBPs associated with steroid hormone receptors are certainly involved in the neurotrophic mechanism of FK506 [10]. To develop FK506 as a novel antifungal or neurotrophic agent, a significant reduction in its immunosuppressive activity is critical.

FK506 is biosynthesized through a hybrid system of a type I modular polyketide synthetase (PKS) and nonribosomal peptide synthetase (NRPS) (Fig. 1). Biosynthesis of FK506 core is initiated by the formation of $(4 R, 5 R)-4,5-$ dihydroxycyclohex-1-enecarboxylic acid (DHCHC), which is derived from the chorismate by chorismatase, which is encoded by $f k b O$ gene in the FK506 biosynthetic gene cluster [13]. The starter unit is extended by ten condensation steps with two malonyl-CoA, two methoxymalonyl-ACP (acyl carrier protein), five methylmalonyl-CoA, and an allymalonyl-CoA/ACP. The products of $t c s A, \operatorname{tcs} B, \operatorname{tcs} C$, and $t c s D$ in the FK506-producing strain are involved in the biosynthesis of the unique extender unit allylmalonyl-CoA. Trans-2-pentenyl-ACP is generated by TcsB ( $\beta$-ketoacyl synthase) and TcsA, which consists of acyl transferase (AT) and ACP didomain, in conjunction with fatty acid synthase pathway. TcsC (crotonyl-CoA carboxylase/reductase) and TcsD (dehydrogenase) catalyze reductive carboxylation reaction and double bond formation, respectively. The 


\section{Tcs A,B,C and D}
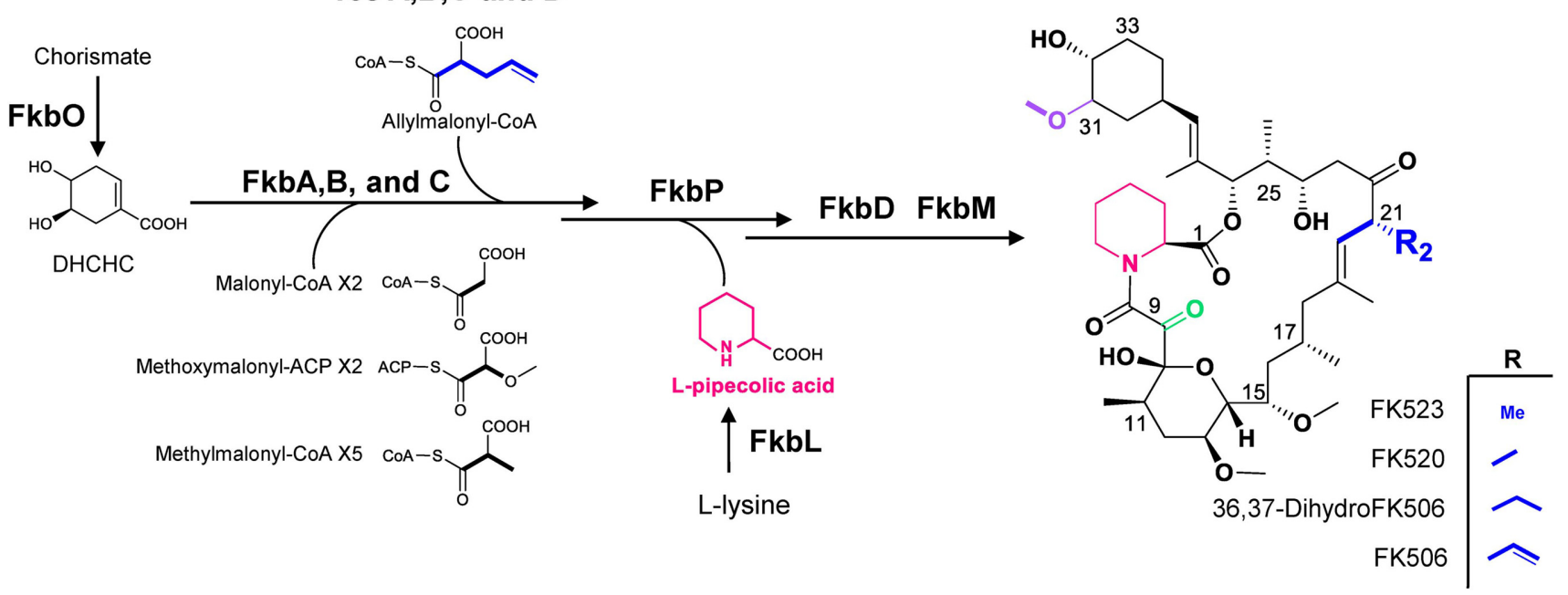

Fig. 1. The biosynthesis of FK506 and its derivatives with modified C21 side chains.

allylmalonyl-CoA/ACP is loaded onto the AT domain of module 4 (AT4) [14]. Particularly, AT4 has broad specificity toward various extender units, resulting in the generation of FK506 derivatives with various C21 side chains. The linear chain obtained by the elongation of PKS is incorporated with L-pipecolate derived from L-lysine by FkbL (lysine cyclodeaminase) [15] and cyclized by NRPS FkbP to form the macrolactone ring. The ring of FK506 requires final post-PKS modification steps, including C9oxidation by FkbD and C31-O-methylation by FkbM [16]. Based on this biosynthetic pathway of FK506, various FK506 derivatives were generated by combinatorial biosynthesis or biosynthetic pathway engineering [4].

Herein, we briefly cover the mode of antifungal and neurotrophic action of FK506. In addition, examples of antifungal and neurotrophic FK506 derivatives with reduced immunosuppressive activity are presented.

\section{Antifungal Mechanism of FK506 against Human Fungal Pathogens}

FK506 exerts antifungal activity and immunosuppressive activity by inhibiting $\mathrm{CaN}$ through the formation of FKBP12-FK506 binary complex [17] (Fig. 2). CaN, the $\mathrm{Ca}^{2+}$ / calmodulin-dependent protein phosphatase, is a heterodimer composed of catalytic A subunit and regulatory B subunit [18]. In addition to its role in T-cell proliferation in humans, it plays a pivotal role in viability, cell wall synthesis, and ion homeostasis in yeast [17-19]. CaN pathway is also essential for the survival of human fungal pathogens, including Cryptococcus neoformans, Candida albicans, and
Aspergillus fumigatus [8]. C. neoformans is the most common cause of major opportunistic infections. The incidence of this infection can lead to cryptococcal meningitis, a common fatal disease [20, 21]. C. albicans is an invasive fungal pathogen, which causes superficial mucosal infection or candidemia due to a bloodstream infection with Candida $[22,23]$. In case of $A$. fumigatus, severe infections, including invasive aspergillosis and severe asthma, occur in immunocompromised patients [24]. It was found that inactivation of $\mathrm{CaN}$ inhibited the growth of human fungal pathogens and abolished the antifungal activity of FK506 $[23,25,26]$.

FK506 has potent antifungal activity against $C$. neoformans at $37^{\circ} \mathrm{C}$. CaN encoded by CNA1 and CNB1 is essential for the virulence of $C$. neoformans [20,25]. Disruption of genes encoding the catalytic A subunit or regulatory B subunit of $\mathrm{CaN}$ in C. neoformans (Cna1 or Cnb1) abolished the growth of $\mathrm{C}$. neoformans at $37^{\circ} \mathrm{C}$ but maintained growth at $24^{\circ} \mathrm{C}[20$, 25]. Unlike C. neoformans, the inhibition of CaN by FK506 in C. albicans is not temperature-sensitive. The C. albicans $\mathrm{CaN}$ mutant strains $\Delta c m p 1$ ( $\Delta c n a 1)$ or $\Delta c n b 1$, which lack catalytic A or regulatory B subunit, respectively, were not defunct at any temperature $[23,27]$. However, $\mathrm{CaN}$ is required for survival in the presence of serum $[23,28]$. The combination of FK506 with fluconazole showed synergistic antifungal activity by inhibiting the virulence of $C$. albicans in serum through the inactivation of $\mathrm{CaN}$ [29]. CaN is of particular importance for filament growth in C. neoformans but not in C. albicans. In case of $A$. fumigatus, FK506 exhibits antifungal activity against $A$. fumigatus regardless of growth temperature, either alone or with caspofungin [30, 31]. In 

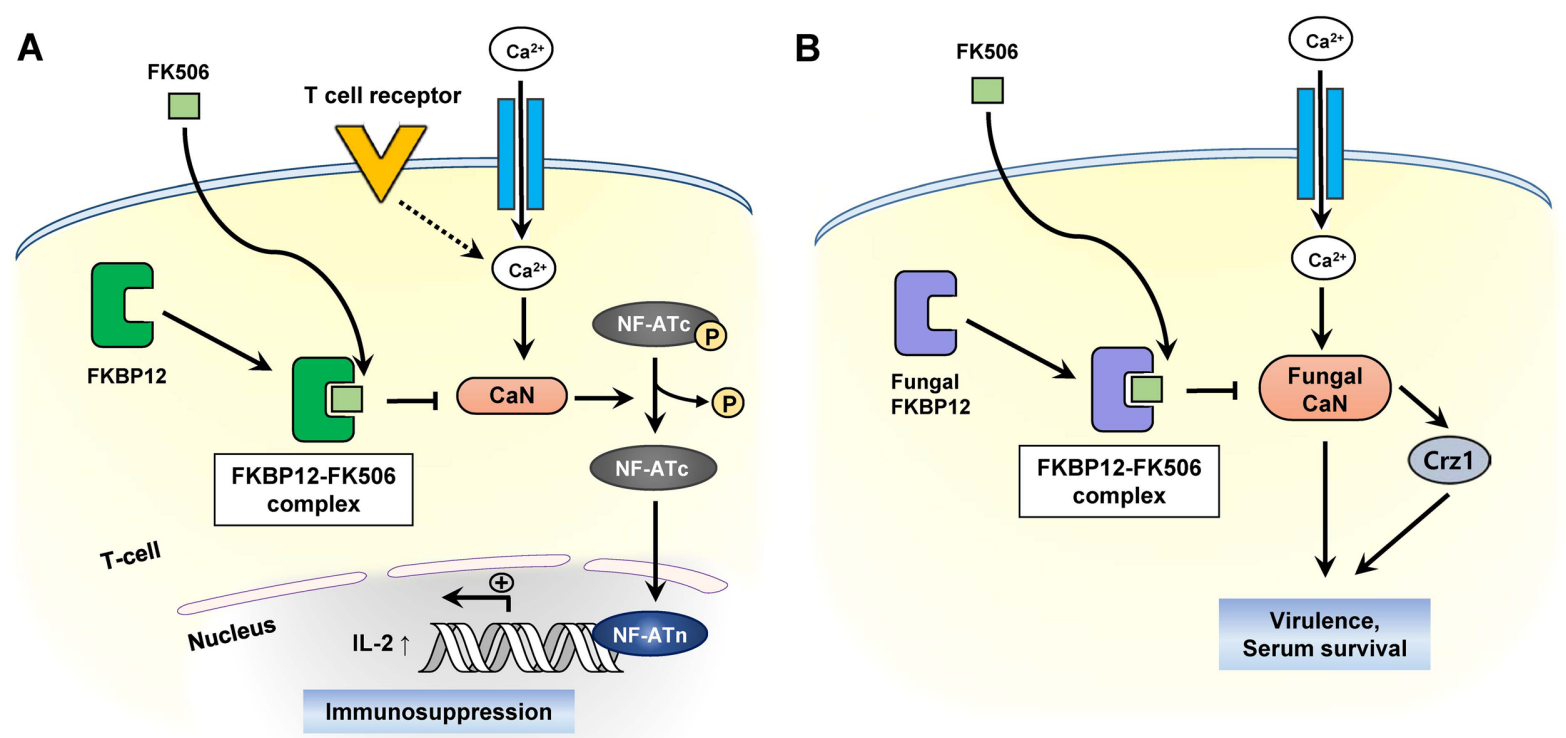

Fig. 2. (A) Mechanism of the immunosuppressive action of FK506. (B) Antifungal action of FK506 against fungal pathogens.

Crz1: Calcineurin-responsive zinc finger 1.

A. fumigatus, CaN plays an important role in hyphal growth [24]. $\Delta$ calA ( $\triangle$ cnaA), A. fumigatus CaN catalytic A subunit mutant, was not affected by various temperatures, and led to decrease filamentation following defective hyphal growth $[8,24,26]$. Taken together, $\mathrm{CaN}$ plays a important role in fungal pathogens, and FK506 exhibits antifungal activity by inhibiting fungal CaNs. Although FK506 has potent antifungal activity against human fungal pathogens, it is necessary to remove its immunosuppressive property to develop FK506 as a new antifungal agent.

\section{Antifungal Activity of FK506 Analogs}

The structure of the human FKBP12-FK506-CaN ternary complex related to immunosuppressive activity was revealed decades ago [32], but the fungal FKBP12-FK506CaN ternary complex has recently been identified [33]. Nevertheless, substantial efforts have been made to develop FK506 analogs that possess reduced immunosuppression but retain potent antifungal activity before the fungal FKBP12-FK506-CaN ternary complexes were identified. L-685,818, which was generated by chemical synthesis, is a less immunosuppressive FK506 analog with antifungal activity (Fig. 3). This analog has hydroxyl group at C18 and ethyl side chain at C21. Due to these differences, L-685,818 showed slightly lower antifungal activity than FK506 against $C$. neoformans but its immunosuppressive activity was significantly reduced ( $10^{5}$-fold reduction) [11]. In addition, it was toxic against $\mathrm{C}$. neoformans at $37^{\circ} \mathrm{C}$ but not at $24^{\circ} \mathrm{C}$, like FK506 [34]. However, L-685,818 did not show antifungal activity against $A$. fumigatus, either alone or with caspofungin [31, 35].

APX879 appended with acetohydrazide at C22 of FK506 is also a chemically modified analog [33] (Fig. 3). This compound also exhibited approximately 71-fold reduced immunosuppression activity compared to FK506 but showed potent antifungal activity against $C$. neoformans, C. albicans, and A. fumigatus. This is because the acetohydrazide chain at C22 of APX879 interacts less favorably with His88 residue of mammalian FKBP12 compared to the corresponding Phe88 of A. fumigatus FKBP12. The in vitro antifungal activity of APX879 against the three fungal pathogens was inferior to that of FK506, but it was less toxic and showed better efficacy than FK506 in the cryptococcal murine model of invasive fungal infection. Furthermore, combination treatment of APX879 + fluconazole was more effective than single treatment of APX879 or fluconazole in the cryptococcal infection murine model. However, the structural complexity of FK506 makes it difficult to chemically synthesize FK506 analogs. Therefore, combinatorial biosynthetic approaches involving the manipulation of FK506 biosynthetic genes could be a viable alternative to generate various biologically improved or modified FK506 analogs.

For recent examples, four FK506 analogs, 9-deoxoprolylFK506 (2), 9-deoxoFK506 (3), 31-O-demethylFK506 (4), and 9-deoxo-31-O-demethylFK506 (5), were generated by modifying post-PKS tailoring genes involved in C9 

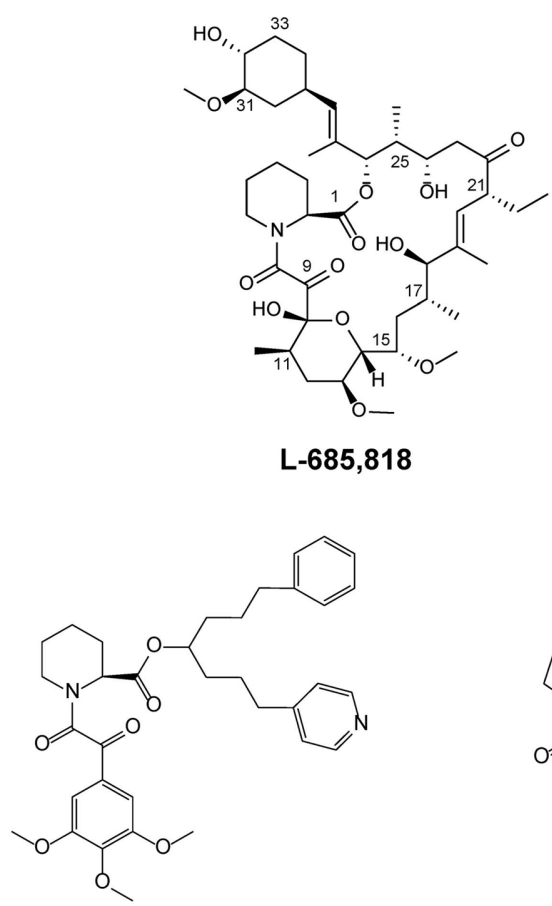

V-10,367
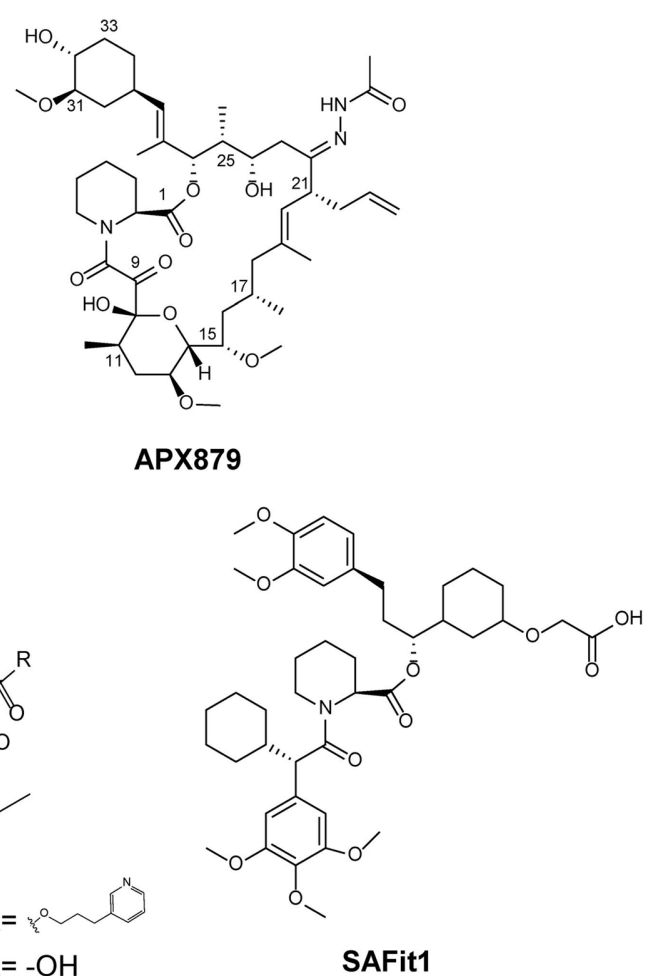

Fig. 3. Non-immunosuppressive FKBP ligands generated by chemical synthesis.

L-685,818, and APX879 exhibited antifungal activity. L-685,818, V-10,367, GPI-1046, GPI-1485, and SAFit1 showed neuronal activities.

oxidation and C31 methylation in Streptomyces sp. KCTC 11604BP [16, 36, 37] (Fig. 4). These compounds were first isolated to elucidate the biosynthetic pathway of FK506; however, they were found to have significantly reduced immunosuppression activity (9.1 to 9937-folds) while retaining potent antifungal activity. Despite lower antifungal activity than that of FK506, all these compounds, except for 9-deoxo-prolylFK506, showed a certain degree of antifungal activity against the aforementioned three major fungal pathogens. In addition, these analogs exhibited synergistic antifungal activity with other commercial antifungal drug fluconazole. Moreover, it was proven that FK506 analogs, like FK506, exerted antifungal effects by inhibiting the CaN pathway because they did not show antifungal activity when the CaN subunit was inactivated in C. neoformans and $C$. albicans. Among the four aforementioned FK506 derivatives, 9-deoxo-31-O-demethylFK506 (5) was selected as a new antifungal candidate on considering both immunosuppressive and antifungal activities, and its in vivo antifungal efficacy was evaluated. Remarkably, combination treatment of 9-deoxo-31-O-demethylFK506 (5) + fluconazole significantly extended the survival of the infected mice, suggesting that $C 9$ and $C 31$ positions in the
FKBP-binding region could be potential sites to control the binding affinity of FK506 to human and fungal FKBP12 [37].

To lower the immunosuppressive activity further while maintaining the antifungal activity, nine FK506 analogs containing modified $\mathrm{C} 1, \mathrm{C} 9, \mathrm{C} 21$, and C31 were recently biosynthesized by combinatorially deleting the allylmalonylCoA synthetic gene $t c s B$ or $t c s D$, which are involved in the formation C21 side chain, in addition to deleting $f k b D$ or $f k b M$, which are involved in the post-PKS modification steps at C9 and C31 positions [38]. Seven compounds, including 9-deoxo-31-O-demethyl-prolylFK506 (6), 9-deoxo36,37-dihydroFK506 (7), 9-deoxoFK523 (9), 31-O-demethyl36,37-dihydroFK506 (10), 9-deoxo-31-O-demethyl-36,37dihydroFK506 (12), 9-deoxo-31-O-demethylFK520 (13), and 9-deoxo-31-O-demethylFK523 (14) were new, and 9deoxoFK520 (8) [39, 40] and 31-O-demethylFK520 (11) [41, 42] were already known (Fig. 4). The structure-activity relationship (SAR) was investigated by assessing immunosuppression and antifungal activity. Compared to 9-deoxo31-O-demethylFK506 (5), C9 and C21 modified analogs generally showed similar or much lower immunosuppressive activity and were more advantageous to maintain strong antifungal activity, except 9-deoxoFK523 (9), which has the 


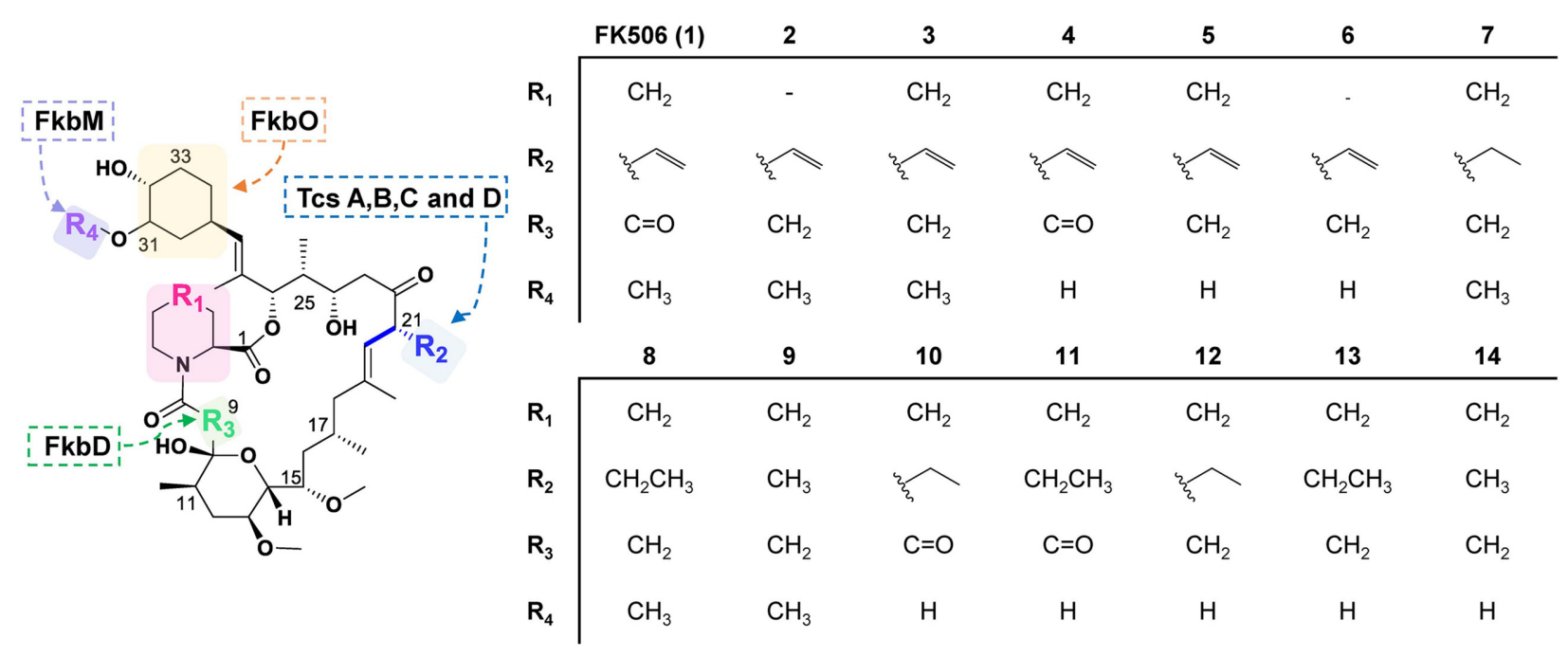

Fig. 4. Representative structures of FK506 derivatives produced by combinatorial biosynthesis.

FK506 derivatives were generated in eight deletion mutants: $\Delta f k b D$ (2 and 3), $\Delta f k b M(4), \Delta f k b D M(\mathbf{5}$ and 6), $\Delta t c s D-\Delta f k b D(7), \Delta t c s B-\Delta f k b D(8$ and 9), $\Delta t c s D-\Delta f k b M$ (10 and 11), $\Delta t c s D-\Delta f k b D M(12)$, and $\Delta t c s B-\Delta f k b D M$ (13 and 14).

methyl side chain at $\mathrm{C} 21$. 31-O-demethyl-36,37-dihydroFK506 (10) and 31-O-demethylFK520 (11), which are modified at both positions of C31 and C21, exhibited high antifungal activity but also showed high immunosuppressive activity. Moreover, C1, C9, C31, and C21 modified analogs showed significantly reduced immunosuppression but no noticeable antifungal activity. Importantly, a combination of fluconazole with FK506 analogs modified with C9 and C21 further enhanced antifungal activity. Consequently, 9-deoxoFK520 (8) has the potential to be a new antifungal agent against a broad range of pathogens [38].

\section{Putative Mechanism of FK506 for Neuroprotection and Neuroregeneration}

FK506 has various neurological activities, such as neuroprotection in focal cerebral ischemia model of stroke [9] and neuroregeneration in animal models of neurodegenerative diseases, including nerve injury [43]. FK506 also enhances neurite outgrowth [10]. The neuronal mechanism of FK506 is unclear, but neuronal properties of FK506 are unequivocally related to FKBP and perhaps even to $\mathrm{CaN}$ in part [44].

The proposed neuroprotective mechanism of FK506 is shown in Fig. 5A [45]. Nerve damage, including cerebral infarction and degenerative disease, is associated with glutamate neurotoxicity, which exhibits toxicity through $\mathrm{N}$-methyl-D-aspartate (NMDA) receptors [46]. However, preventing nitric oxide $(\mathrm{NO})$ production associated with NMDA toxicity may have a protective effect against this neurotoxicity [47]. NO synthase (NOS) can be phosphorylated by inhibiting $\mathrm{CaN}$, thus preventing the formation of $\mathrm{NO}$ $[48,49]$. Therefore, the FK506-FKBP complex exhibits neuroprotective activity by inhibiting $\mathrm{CaN}$, dephosphorylation of NOS, and reduction of NO formation against glutamate toxicity in vitro [49]. Nonetheless, it is believed that an alternative mechanism may be involved in its neuroprotective effects because it was observed that $\mathrm{NO}$ inhibitors were not neuroprotective in vivo [50].

Regarding nerve regeneration activity, it has been reported that FKBP52 rather than FKBP12 is involved in the neuroregenerative action of FK506 [51, 52]. In neuronal hippocampal cultures, FK506 treatment showed comparable neuronal growth in FKBP12 knockout and wild-type mice ( $30 \%$ and $26 \%$, respectively). In addition, it was confirmed that the FKBP52 antibody, which does not bind FKBP12, interferes with neurite outgrowth of FK506 [51]. Putative neuroregenerative action begins with the dissociation of steroid receptor complex comprising the steroid receptor protein, FKBP52, Hsp90, and p23 [53, 54] (Fig. 5B). FK506 binds to FKBP52 to dissociate the complex, which activates Hsp90 to release p23. Hsp90 stimulates nerve regeneration by activating the mitogen-associated protein (MAP) kinase pathway and then increases c-Jun and GAP-43 involved in nerve regeneration by the ERK pathway [55, 56]. Nerve growth factor (NGF) also synergistically activates the MAP kinase pathway along with FK506 to promote nerve regeneration [10]. P23 promotes nerve regeneration by the ERK pathway or unknown pathways. In summary, the 
A

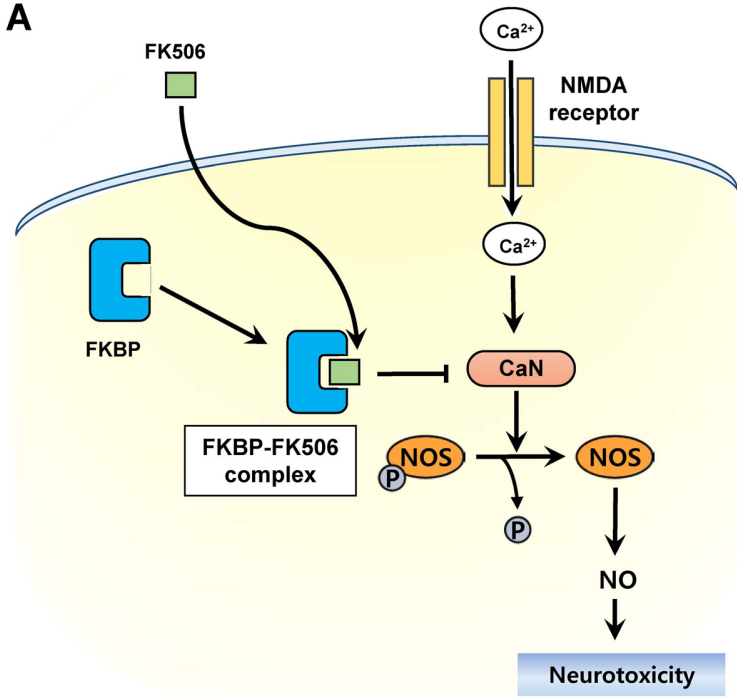

B

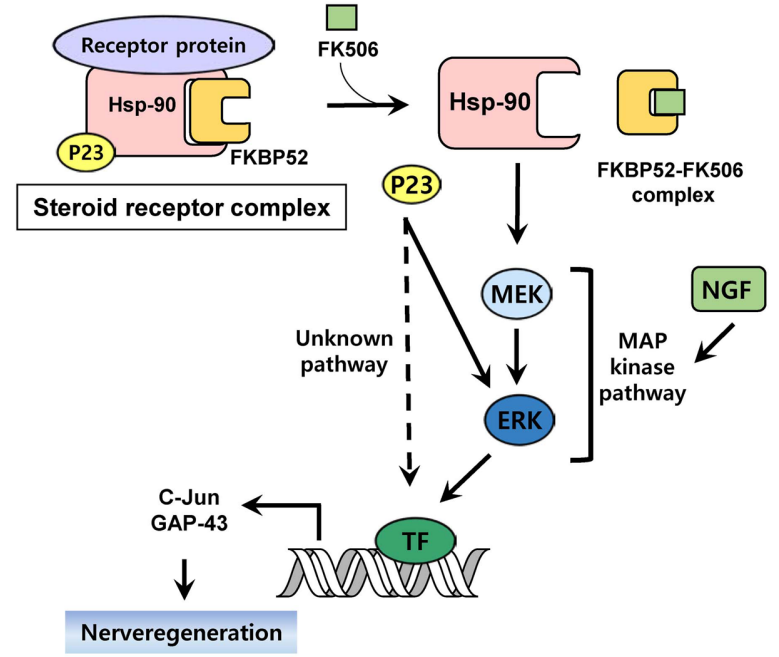

Fig. 5. Proposed neurotrophic mechanism of action of FK506.

FK506 is involved in neuroprotective (A) and neuroregenerative action (B).

FKBP52-FK506 complex mediates nerve regeneration by the activation of Hsp90 and dissociation of p23 [51-53].

In addition, FKBP51, which is structurally very similar to FKBP52, is recently known to be involved in neuroregenerative activity [57-59]. It has been discovered that selective inhibition of FKBP51 can enhance neuroregeneration [59]. Notably, these two proteins have opposite effects on steroid hormone receptors [60,61]. Therefore, it is important to develop FK506 derivatives that can distinguish between FKBP51 and FKBP52. Furthermore, FKBP52 may cause reproductive problems, but FKBP51 may be effective in treating depression [62-64]. FKBP51 and FKBP52 have similar affinity to FK506 [65, 66], high homology [67, 68], and similar active sites [69]; thus, it is difficult to identify the true target of the non-immunosuppressive FKBP ligands developed before 2014 [70, 71].

\section{FK506 Analogs with Neuronal Properties}

It has been reported that immunosuppressive activity of FK506 can be separated from its neuronal activity [72]. The chemically synthesized V-10,367, which lacks the right side of FK506, is a representative FKBP12 inhibitor with significantly reduced immunosuppressive activity [73] (Fig. 3). It has shown neuroprotective activity in NTPmethyl-4-phenyl-1,2,3,6-tetrahydropyridine (MPTP)-treated mouse and rat models of Parkinson's disease [74] and has shown functional recovery, neuronal regeneration, and neurite growth activity [72]. L-685,818, mentioned in the previous antifungal section, has also showed neuronal regeneration activity [75]. GPI-1046, which has $>5000$-foldreduced immunosuppression activity compared to FK506, has exhibited neuronal properties, including neuroprotection, neurite outgrowth, and nerve recovery, and has become a clinical candidate for the treatment of Parkinson's disease [76, 77] (Fig. 3). Since then, several studies have suggested FKBP52 as the target of neuronal regeneration [51, 52, 77], and GPI-1485, one of the GPI-1046 series, was studied up to phase 2 as a non-FKBP12 inhibitor but failed due to lack of clinical benefit [57, 78, 79] (Fig. 3). Nevertheless, the development of FKBP inhibitors is ongoing, and as mentioned above, it has become particularly important to distinguish between FKBP51 and FKBP52 as neuronal regeneration targets. The recently synthesized SAFit1 targets only FKBP51, did not affect the production of IL-2, which causes an immune response, and promoted neurite growth [70] (Fig. 3).

FK506 derivatives with reduced immunosuppressive and neuronal regenerative activities have also been developed by the modification of the FK506 biosynthetic pathway. For example, new FK506 derivatives were generated by mutational biosynthesis. 36-MethylFK506 (15) and 36fluoroFK520 (16) were produced when 4-methylpentanoic acid and 4-fluorocrotonic acid were fed to the $t c s B$ mutant, respectively (Fig. 6A). Both these substances showed neurite outgrowth, albeit with slightly reduced immunosuppressive activity. In particular, neurite outgrowth of 36-methylFK506 (15) increased 1.2-fold compared to FK506 [14]. 32- 
A<smiles>CC(C)CCC(=O)O</smiles>

4-methylphenic acid<smiles>O=C(O)/C=C/CF</smiles>

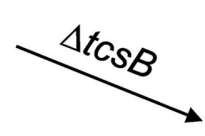

4-fluorocrotonic acid

B

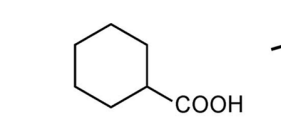

3-methoxycyclohexane1-carboxylic acid

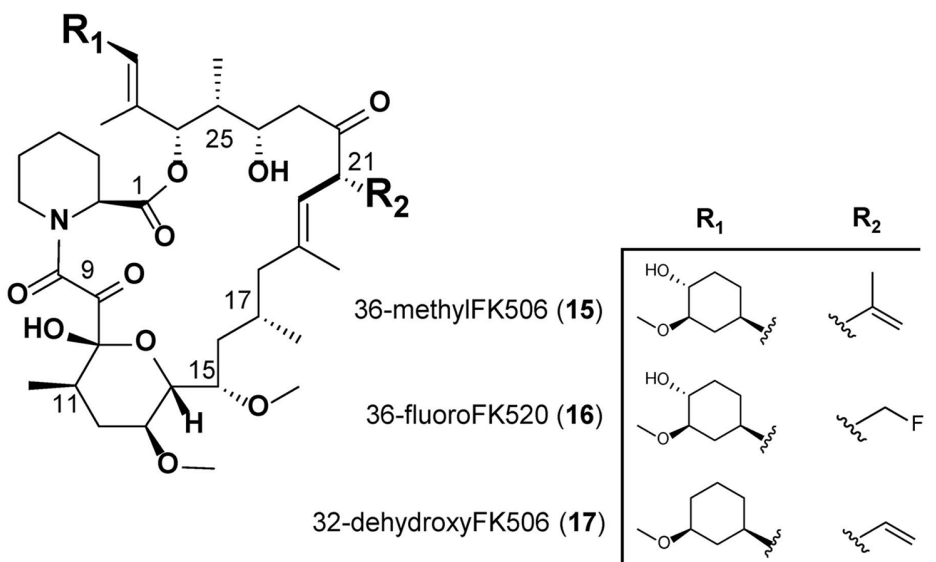

Fig. 6. Mutasynthesis of FK506 derivatives in $\triangle t c s B$ or $\Delta f k b O$ strain with chemical feeding.

(A) 36-methyl-FK506 (15) and 36-fluoro-FK520 (16) were obtained from the culture of $\Delta t c s B$ strain supplemented with 4-methylphenic acid and 4fluorocrotonic acid, respectively. (B) 32-dehydroxy-FK506 (17) was obtained from the culture of $\Delta f k b O$ strain supplemented with 3methoxycyclohexane-1-carboxylic acid.

DehydroxyFK506 (17) is also an FK506 derivative produced by feeding 3-methoxycyclohexane-1-carboxylic acid to the deletion mutant of $f k b O$, which is involved in the biosynthesis of the starter unit of FK506. This compound reportedly shows reduced immunosuppression by threefold and slightly increased neurite outgrowth activity than FK506 [80] (Fig. 6B). 9-Deoxo-prolylFK506 (2), which was obtained through the deletion of $f k b D$, exhibited slightly reduced neuronal regeneration activity but significantly reduced immunosuppression [36] (Fig. 4).

\section{Summary and Future Perspectives}

The strong immunosuppressive activity of FK506 should be reduced or eliminated to harness the other useful effects, such as antifungal and neurotrophic activities. Several studies have shown that these activities can be separated from immunosuppressive activity and maintained. FKBP12 and $\mathrm{CaN}$ were found to be important targets for immunosuppressive and antifungal activity of FK506 [8], and chemically synthesized FK506 derivatives showed the possibility of separation of immunosuppressive and antifungal activities [11, 33]. In addition, various FK506 derivatives were developed through combinatorial biosynthesis and showed the possibility of modulating the immunosuppressive and antifungal activity by modifying some specific functional groups of FK506 [37, 38]. The structure of fungal FKBP12-FK506-CaN, which has been recently discovered, will provide a valuable insight to structure-guided fungal-specific inhibitor design [33].

The mechanisms of neuroprotective and neuronal regeneration activity of FK506 are unclear. In the early studies, chemically synthesized FK506 derivatives showed that neuronal activity could be separated from immunosuppressive activity $[73,76]$ and that neuronal regeneration was related to FKBP52 as they exhibited neuronal regeneration property without binding to FKBP12 [51, 52]. FKBP51 was recently suggested to be a true target for neuronal regeneration. Moreover, while FKBP52 may cause reproductive function [62, 63], FKBP51 is a safe target for neuronal regeneration and stress-related disorders, such as depression [58, 64]. Although these two proteins were difficult to distinguish due to their common features, SAFit derivatives showed the possibility of distinguishing these two proteins [70]. FK506 derivatives made to date through biosynthesis showed significantly reduced immunosuppression while maintaining comparable neuroregenerative activities as FK506. However, it is not certain if these derivatives can distinguish between FKBP51 and FKBP52 [14, 36, 80]. Therefore, considering the advantages of combinatorial biosynthesis, which enables biosynthesis of diverse structures, detailed SAR and target identification studies of various derivatives in the future are expected to increase the possibility to develop a new therapeutic agent for neurological diseases. 


\section{Acknowledgments}

This work was supported by National Research Foundation of Korea (NRF) grants funded by the Ministry of Science and ICT (MSIT) (2019R1A2B5B03069338), the Bio \& Medical Technology Development Program of the NRF funded by the MSIT (2018M3A9F3079662), the Collaborative Genome Program of the Korea Institute of Marine Science and Technology Promotion (KIMST) (No. 20180430), and a grant of the Korea Health Technology R\&D Project through the Korea Health Industry Development Institute (KHIDI) funded by the Ministry of Health \& Welfare (HI18C1664).

\section{Conflict of Interest}

The authors have no financial conflicts of interest to declare.

\section{References}

1. Kino $T$, Hatanaka $H$, Miyata $S$, Inamura $N$, Nishiyama $M$, Yajima T, et al. 1987. FK-506, a novel immunosuppressant isolated from a Streptomyces. II. Immunosuppressive effect of FK-506 in vitro. J. Antibiot. 40: 1256-1265.

2. Parsons WH, Sigal NH, Wyvratt MJ. 1993. FK-506--a novel immunosuppressant. Ann. NY Acad. Sci. 685: 22-36.

3. Kino T, Hatanaka $H$, Hashimoto M, Nishiyama M, Goto T, Okuhara M, et al. 1987. FK-506, a novel immunosuppressant isolated from a Streptomyces. I. Fermentation, isolation, and physico-chemical and biological characteristics. J. Antibiot. 40: $1249-1255$

4. Ban YH, Park SR, Yoon YJ. 2016. The biosynthetic pathway of FK506 and its engineering: from past achievements to future prospects. J. Ind. Microbiol. Biotechnol. 43: 389-400.

5. Barreiro C, Martínez-Castro M. 2014. Trends in the biosynthesis and production of the immunosuppressant tacrolimus (FK506). Appl. Microbiol. Biotechnol. 98: 497-507.

6. Mo S, Lee SK, Jin YY, Suh JW. 2016. Improvement of FK506 production in the high-yielding strain Streptomyces sp. RM7011 by engineering the supply of allylmalonyl-CoA through a combination of genetic and chemical approach. J. Microbiol. Biotechnol. 26: 233-40.

7. Nakagawa H, Etoh T, Yokota Y, Ikeda F, Hatano K, Teratani $\mathrm{N}$, et al. 1996. Tacrolimus has antifungal activities against Malassezia furfur isolated from healthy adults and patients with atopic dermatitis. Clin. Drug. Invest. 12: 244-250.

8. Steinbach WJ, Reedy JL, Cramer RA Jr, Perfect JR, Heitman J. 2007. Harnessing calcineurin as a novel anti-infective agent against invasive fungal infections. Nat. Rev. Microbiol. 5: 418-430.

9. Sharkey J, Butcher SP. 1994. Immunophilins mediate the neuroprotective effects of FK506 in focal cerebral ischaemia. Nature 371: 336-339.
10. Lyons WE, George EB, Dawson TM, Steiner JP, Snyder SH. 1994. Immunosuppressant FK506 promotes neurite outgrowth in cultures of PC12 cells and sensory ganglia. Proc. Natl. Acad. Sci. USA 91: 3191-3195.

11. Dumont FJ, Staruch MJ, Koprak SL, Siekierka JJ, Lin CS, Harrison R, et al. 1992. The immunosuppressive and toxic effects of FK-506 are mechanistically related: pharmacology of a novel antagonist of FK-506 and rapamycin. J. Exp. Med. 176: 751-760.

12. Liu J, Farmer JD Jr, Lane WS, Friedman J, Weissman I, Schreiber SL. 1991. Calcineurin is a common target of cyclophilin-cyclosporin A and FKBP-FK506 complexes. Cell 66: 807-815.

13. Andexer JN, Kendrew SG, Nur-e-Alam M, Lazos O, Foster TA, Zimmermann AS, et al. 2011. Biosynthesis of the immunosuppressants FK506, FK520, and rapamycin involves a previously undescribed family of enzymes acting on chorismate. Proc. Natl. Acad. Sci. USA 108: 4776-4781.

14. Mo S, Kim DH, Lee JH, Park JW, Basnet DB, Ban YH, et al. 2011. Biosynthesis of the allylmalonyl-CoA extender unit for the FK506 polyketide synthase proceeds through a dedicated polyketide synthase and facilitates the mutasynthesis of analogues. J. Am. Chem. Soc. 133: 976-985.

15. Motamedi H, Shafiee A. 1998. The biosynthetic gene cluster for the macrolactone ring of the immunosuppressant FK506. Eur. J. Biochem. 256: 528-534.

16. Ban YH, Shinde PB, Hwang JY, Song MC, Kim DH, Lim SK, et al. 2013. Characterization of FK506 biosynthetic intermediates involved in post-PKS elaboration. J. Nat. Prod. 76: 1091-1098.

17. Breuder T, Hemenway CS, Movva NR, Cardenas ME, Heitman J. 1994. Calcineurin is essential in cyclosporin Aand FK506-sensitive yeast strains. Proc. Natl. Acad. Sci. USA 91: 5372-5376.

18. Watanabe Y, Perrino BA, Chang BH, Soderling TR. 1995. Identification in the calcineurin A subunit of the domain that binds the regulatory B subunit. J. Biol. Chem. 270: 456-460.

19. Withee JL, Mulholland J, Jeng R, Cyert MS. 1997. An Essential role of the yeast pheromone-induced $\mathrm{Ca}^{2+}$ signal is to activate calcineurin. Mol. Biol. Cell 8: 263-277.

20. Odom A, Muir S, Lim E, Toffaletti DL, Perfect J, Heitman J. 1997. Calcineurin is required for virulence of Cryptococcus neoformans. EMBO J. 16: 2576-2589.

21. Mitchell TG, Perfect JR. 1995. Cryptococcosis in the era of AIDS-100 years after the discovery of Cryptococcus neoformans. Clin. Microbiol. Rev. 8: 515-548.

22. Berman J, Sudbery PE. 2002. Candida albicans: a molecular revolution built on lessons from budding yeast. Nat. Rev. Genet. 3: 918-930.

23. Blankenship JR, Wormley FL, Boyce MK, Schell WA, Filler SG, Perfect JR, et al. 2003. Calcineurin is essential for Candida albicans survival in serum and virulence. Eukaryot. Cell 2: 422-430. 
24. Steinbach WJ, Cramer RA Jr, Perfect BZ, Asfaw YG, Sauer TC, Najvar LK, et al. 2006. Calcineurin controls growth, morphology, and pathogenicity in Aspergillus fumigatus. Eukaryot. Cell 5: 1091-1103.

25. Fox DS, Cruz MC, Sia RA, Ke H, Cox GM, Cardenas ME, et al. 2001. Calcineurin regulatory subunit is essential for virulence and mediates interactions with FKBP12-FK506 in Cryptococcus neoformans. Mol. Microbiol. 39: 835-849.

26. da Silva Ferreira ME, Heinekamp T, Härtl A, Brakhage AA, Semighini CP, Harris SD, et al. 2007. Functional characterization of the Aspergillus fumigatus calcineurin. Fungal Genet. Biol. 44: 219-230.

27. Bader T, Bodendorfer B, Schröppel K, Morschhäuser J. 2003. Calcineurin is essential for virulence in Candida albicans. Infect. Immun. 71: 5344-5354.

28. Blankenship JR, Heitman J. 2005. Calcineurin is required for Candida albicans to survive calcium stress in serum. Infect. Immun. 73: 5767-5774.

29. Cruz MC, Goldstein AL, Blankenship JR, Del Poeta M, Davis D, Cardenas ME, et al. 2002. Calcineurin is essential for survival during membrane stress in Candida albicans. EMBO J. 21: 546-559.

30. High KP. 1994. The antimicrobial activities of cyclosporine, FK506, and rapamycin. Transplantation 57: 1689-1700.

31. Steinbach WJ, Schell WA, Blankenship JR, Onyewu C, Heitman J, Perfect JR. 2004. In vitro interactions between antifungals and immunosuppressants against Aspergillus fumigatus. Antimicrob. Agents. Chemother. 48: 1664-1669.

32. Griffith JP, Kim JL, Kim EE, Sintchak MD, Thomson JA, Fitzgibbon MJ, et al. 1995. X-ray structure of calcineurin inhibited by the immunophilin-immunosuppressant FKBP12FK506 complex. Cell 82: 507-522.

33. Juvvadi PR, Fox D 3rd, Bobay BG, Hoy MJ, Gobeil SMC, Venters RA, et al. 2019. Harnessing calcineurin-FK506FKBP12 crystal structures from invasive fungal pathogens to develop antifungal agents. Nat. Commun. 10: 4275.

34. Odom A, Del Poeta M, Perfect J, Heitman J. 1997. The immunosuppressant FK506 and its nonimmunosuppressive analog L-685,818 are toxic to Cryptococcus neoformans by inhibition of a common target protein. Antimicrob. Agents. Chemother. 41: 156-161.

35. Kontoyiannis DP, Lewis RE, Osherov N, Albert ND, May GS. 2003. Combination of caspofungin with inhibitors of the calcineurin pathway attenuates growth in vitro in Aspergillus species. J. Antimicrob. Chemother. 51: 313-316.

36. Shinde PB, Ban YH, Hwang JY, Cho Y, Chen YA, Cheong E, et al. 2015. A non-immunosuppressive FK506 analogue with neuroregenerative activity produced from a genetically engineered Streptomyces strain. RSC Adv. 5: 6823-6828.

37. Lee Y, Lee KT, Lee SJ, Beom JY, Hwangbo A, Jung JA, et al. 2018. In vitro and in vivo assessment of FK506 analogs as novel antifungal drug candidates. Antimicrob. Agents Chemother. 62: $\mathrm{e} 01627-18$.
38. Beom JY, Jung JA, Lee KT, Hwangbo A, Song MC, Lee Y, et al. 2019. Biosynthesis of nonimmunosuppressive FK506 analogues with antifungal activity. J. Nat. Prod. 82: 2078-2086.

39. Cullen WP, Guadliana MA, Huang LH, Kaneda K, Kojima N, Kojima Y, et al. 1992. Novel immunosuppressant agent from Streptomyces braegensis. WO Patent 1992/018506.

40. Baumann K, Knapp H, Strnadt G, Schulz G, Grassberger MA. 1999. Carbonyl to methylene conversions at the tricarbonyl-portion of ascomycin derivatives. Tetrahedron Lett. 40: 7761-7764.

41. Chen TS, Arison BH, Wicker LS, Inamine ES, Monaghan RL. 1992. Microbial transformation of immunosuppressive compounds. I. Desmethylation of FK506 and immunomycin (FR 900520) by Actinoplanes sp. ATCC 53771. J. Antibiot. 45: 118-123.

42. Brizuela L, Chrebet G, Bostian KA, Parent SA. 1991. Antifungal properties of the immunosuppressant FK-506: identification of an FK-506-responsive yeast gene distinct from FKB1. Mol. Cell. Biol. 11: 4616-4626.

43. Gold BG, Storm-Dickerson T, Austin DR. 1994. The immunosuppressant FK506 increases functional recovery and nerve regeneration following peripheral nerve injury. Restor. Neurol. Neurosci. 6: 287-296.

44. Steiner JP, Dawson TM, Fotuhi M, Glatt CE, Snowman AM, Cohen $\mathrm{N}$, et al. 1992. High brain densities of the immunophilin FKBP colocalized with calcineurin. Nature 358: 584-587.

45. Snyder SH, Sabatini DM. 1995. Immunophilins and the nervous system. Nat. Med. 1: 32-37.

46. Choi DW. 1988. Glutamate neurotoxicity and diseases of the nervous system. Neuron 1: 623-634.

47. Dawson VL, Dawson TM, Bartley DA, Uhl GR, Snyder SH. 1993. Mechanisms of nitric oxide-mediated neurotoxicity in primary brain cultures. J. Neurosci. 13: 2651-2661.

48. Conde M, Andrade J, Bedoya FJ, Santa Maria C, Sobrino F. 1995. Inhibitory effect of cyclosporin A and FK506 on nitric oxide production by cultured macrophages. Evidence of a direct effect on nitric oxide synthase activity. Immunology 84: 476-481.

49. Dawson TM, Steiner JP, Dawson VL, Dinerman JL, Uhl GR, Snyder SH. 1993. Immunosuppressant FK506 enhances phosphorylation of nitric oxide synthase and protects against glutamate neurotoxicity. Proc. Natl. Acad. Sci. USA 90: 9808-9812.

50. Butcher SP, Henshall DC, Teramura Y, Iwasaki K, Sharkey J. 1997. Neuroprotective actions of FK506 in experimental stroke: in vivo evidence against an antiexcitotoxic mechanism. $J$. Neurosci. 17: 6939-6946.

51. Gold BG, Densmore V, Shou W, Matzuk MM, Gordon HS. 1999. Immunophilin FK506-binding protein 52 (not FK506binding protein 12) mediates the neurotrophic action of FK506. J. Pharmacol. Exp. Ther. 289: 1202-1210.

52. Gold BG. 1999. FK506 and the role of the immunophilin FKBP-52 in nerve regeneration. Drug Metab. Rev. 31: 649-663. 
53. Gold BG, Villafranca JE. 2003. Neuroimmunophilin Ligands: The development of novel neuroregenerative/neuroprotective compounds. Curr. Top. Med. Chem. 3: 1368-1375.

54. Pratt WB, Toft DO. 1997. Steroid receptor interactions with heat shock protein and immunophilin chaperones. Endocr. Rev. 18: 306-360.

55. Sétáló G Jr, Singh M, Guan X, Toran-Allerand CD. 2002. Estradiol-induced phosphorylation of ERK1/2 in explants of the mouse cerebral cortex: the roles of heat shock protein 90 (Hsp90) and MEK2. J. Neurobiol. 50: 1-12.

56. Herdegen T, Skene P, Bähr M. 1997. The c-jun transcription factor -bipotential mediator of neuronal death, survival and regeneration. Trend Neurosci. 20: 227-231.

57. Hausch F. 2015. FKBPs and their role in neuronal signaling. Biochim. Biophys. Acta 1850: 2035-2040.

58. Schmidt MV, Paez-Pereda M, Holsboer F, Hausch F. 2012. The prospect of FKBP51 as a drug target. ChemMedChem 7: 1351-1359.

59. Quintá HR, Maschi D, Gomez-Sanchez C, Piwien-Pilipuk G, Galigniana MD. 2010. Subcellular rearrangement of hsp90binding immunophilins accompanies neuronal differentiation and neurite outgrowth. J. Neurochem. 115: 716-734.

60. Cheung-Flynn J, Prapapanich V, Cox MB, Riggs DL, SuarezQuian C, Smith DF. 2005. Physiological role for the cochaperone FKBP52 in androgen receptor signaling. Mol. Endocrinol. 19: 1654-1666.

61. Yong W, Yang Z, Periyasamy S, Chen H, Yucel S, Li W, et al. 2007. Essential role for co-chaperone Fkbp52 but not Fkbp51 in androgen receptor-mediated signaling and physiology. J. Biol. Chem. 282: 5026-5036.

62. Tranguch S, Cheung-Flynn J, Daikoku T, Prapapanich V, Cox MB, Xie $\mathrm{H}$, et al. 2005. Cochaperone immunophilin FKBP52 is critical to uterine receptivity for embryo implantation. Proc. Natl. Acad. Sci. USA 102: 14326-14331.

63. Yang Z, Wolf IM, Chen H, Periyasamy S, Chen Z, Yong W, et al. 2006. FK506-Binding protein 52 is essential to uterine reproductive physiology controlled by the progesterone receptor A isoform. Mol. Endocrinol. 20: 2682-2694.

64. Hartmann J, Wagner KV, Liebl C, Scharf SH, Wang XD, Wolf $\mathrm{M}$, et al. 2012. The involvement of FK506-binding protein 51 (FKBP5) in the behavioral and neuroendocrine effects of chronic social defeat stress. Neuropharmacology 62: 332-339.

65. Kozany C, März A, Kress C, Hausch F. 2009. Fluorescent probes to characterise FK506-binding proteins. Chembiochem 10: $1402-1410$.

66. Gopalakrishnan R, Kozany C, Wang Y, Schneider S, Hoogeland B, Bracher A, et al. 2012. Exploration of pipecolate sulfonamides as binders of the FK506-binding proteins 51 and 52. J. Med. Chem. 55: 4123-4131.

67. Sinars CR, Cheung-Flynn J, Rimerman RA, Scammell JG, Smith DF, Clardy J. 2003. Structure of the large FK506- binding protein FKBP51, an Hsp90-binding protein and a component of steroid receptor complexes. Proc. Natl. Acad. Sci. USA 100: 868-873.

68. Blackburn EA, Walkinshaw MD. 2011. Targeting FKBP isoforms with small-molecule ligands. Curr. Opin. Pharmacol. 11: 365-371.

69. Bracher A, Kozany C, Hähle A, Wild P, Zacharias M, Hausch F. 2013. Crystal structures of the free and ligandbound FK1-FK2 domain segment of FKBP52 reveal a flexible inter-domain hinge. J. Mol. Biol. 425: 4134-4144.

70. Gaali S, Kirschner A, Cuboni S, Hartmann J, Kozany C, Balsevich G, et al. 2015. Selective inhibitors of the FK506binding protein 51 by induced fit. Nat. Chem. Biol. 11: 33-37.

71. Feng X, Sippel C, Bracher A, Hausch F. 2015. StructureAffinity Relationship Analysis of Selective FKBP51 Ligands. J. Med. Chem. 58: 7796-7806.

72. Gold BG, Zeleny-Pooley M, Wang MS, Chaturvedi P, Armistead DM. 1997. A nonimmunosuppressant FKBP-12 ligand increases nerve regeneration. Exp. Neurol. 147: 269-278.

73. Armistead DM, Badia MC, Deininger DD, Duffy JP, Saunders JO, Tung RD, et al. 1995. Design, synthesis and structure of non-macrocyclic inhibitors of FKBP12, the major binding protein for the immunosuppressant FK506. Acta. Crystallogr. D 51: 522-528.

74. Costantini LC, Cole D, Chaturvedi P, Isacson O. 2001. Immunophilin ligands can prevent progressive dopaminergic degeneration in animal models of Parkinson's disease. Eur. J. Neurosci. 13: 1085-1092.

75. Steiner JP, Connolly MA, Valentine HL, Hamilton GS, Dawson TM, Hester L, et al. 1997. Neurotrophic actions of nonimmunosuppressive analogues of immunosuppressive drugs FK506, rapamycin and cyclosporin A. Nat. Med. 3: 421-428.

76. Steiner JP, Hamilton GS, Ross DT, Valentine HL, Guo H, Connolly MA, et al. 1997. Neurotrophic immunophilin ligands stimulate structural and functional recovery in neurodegenerative animal models. Proc. Natl. Acad. Sci. USA 94: 2019-2024.

77. Ruan B, Pong K, Jow F, Bowlby M, Crozier RA, Liu D, et al. 2008. Binding of rapamycin analogs to calcium channels and FKBP52 contributes to their neuroprotective activities. Proc. Natl. Acad. Sci. USA 105: 33-38.

78. Ravina BM, Fagan SC, Hart RG, Hovinga CA, Murphy DD, Dawson TM, et al. 2003. Neuroprotective agents for clinical trials in Parkinson's disease: a systematic assessment. Neurology 60: 1234-1240.

79. NINDS NET-PD Investigators. 2007. A randomized clinical trial of coenzyme Q10 and GPI-1485 in early Parkinson disease. Neurology 68: 20-28.

80. Ban YH, Lee JH, Gu GR, Lee B, Mo S, Kwon HJ, et al. 2013. Mutational biosynthesis of a FK506 analogue containing a non-natural starter unit. Mol. Biosyst. 9: 944-947. 